\title{
IDENTIFICATION OF LAND COVER ALTERATIONS IN THE ALTA MURGIA NATIONAL PARK (ITALY) WITH VHR SATELLITE IMAGERY
}

\author{
M. CAPRIOLI \& E. TARANTINO
}

Polytechnic University of Bari, Italy.

\section{ABSTRACT}

Land cover exerts a great influence on many basic environmental processes and consequently any transformation in it may have a marked impact on the environment from the local to the global scales. In multidisciplinary research contexts, satellite remote sensing offers opportunities both to evaluate the effects of these processes and to provide one of the information layers needed for designing national strategies oriented to protection and sustainable use of our resources. The advent of recent satellite imagery has increased the possibility to investigate large-scale areas in great detail. Together with an increase in spatial and radiometric resolution, there is, usually, an increase in the variability within land parcels, generating a decrease in the accuracy of land use classification on a per-pixel basis. In order to avoid such negative impacts, an object-oriented classification methodology on IKONOS multispectral data has been implemented on the test area of the Alta Murgia National Park, in the Apulia region (Italy), where soil adaptation for agricultural practices, through rock breaking, has taken place over the last 20 years. The analysis has been conducted with a classification strategy that is able to distinguish land use functions on the basis of differences in spatial distribution and pattern of land cover forms. It consists of two phases: segmentation of the image into meaningful multipixel objects of various sizes, based on both spectral and spatial characteristics of groups of pixels; then, assignment of the segments (objects) to classes using fuzzy logic and a hierarchical decision key.

Keywords: land cover transformation, object-oriented classification, VHR satellite imagery.

\section{INTRODUCTION}

Desertification is an ever-increasing problem in the Mediterranean basin countries, threatening 27\% of the Italian territory. It stems principally from the seasonal extremes of climate and the geological nature of areas [1]. This process is accelerated by forest destruction, water pollution, wind and water erosion, salinization and inadequate soil management under both cultivated and uncultivated regimes. One of the major problems affecting the soil is the severity with which the degradation processes reduce its biological potential. An unsustainable, rapid reduction, which cannot be mitigated by using suitable mechanisms, leads consequently to desertification [2]. The United Nations Environmental Programme (UNEP) has continuously redefined the concept of desertification over the past 20 years. In fact, at the end of the 1970's, the UNEP defined this concept as 'reduction or destruction of soil biological potential is conducive to conditions of desertification' [3]. In the early 1990s, they defined it as 'land degradation in arid, semi-arid and dry subhumid areas resulting from various factors, including climatic variations and human activities' [4].

In the Apulia region, and particularly in the Alta Murgia area, the rock-breaking activity must be added to the list of land degradation causes. The custom of removing stones has been practiced for a long time. It consists in removing outcrops of stones for rural constructions from cultivated fields at the end of every productive cycle. In recent years, such a practice has been substituted by breaking rocks, which consists in deep ploughing and the mechanical crushing of rocks to transform pastureland into cultivated land. This custom was legally permitted by regional laws and was encouraged with public loans. It spread all over the Murgia area, destroying thousands of hectares of pastureland and endangering a fragile ecosystem. 
Today, there are no longer any public loans, but the practice, although much less now, still continues and the possibility is that the rehabilitation of such areas would require a long time. The effects of this practice, which emerged during the period of the constitution of Alta Murgia National Park (Fig. 1), have been numerous (e.g. the destruction of the signs of a material rural culture and the disappearance of pseudo-steppe vegetation with the consequent death of animal species living in this environment). This park was designated in 1998 by the national government of Italy (Ministry of Environment) and finally created in 2002 [5] and extends over a territory between the Bradanica Valley and the depressions toward the Adriatic Coast, with 13 towns collocated along its perimeter. The presence in Alta Murgia of an almost deserted large hilly area - very rare in Italy - full of antique farmhouses (built for pastoral agriculture along ancient and even Roman roads), surrounded by important historical settlements and cities and with extraordinary natural and artificial remains (the Dinosaur Valley and the archaeological settlements in Altamura, the mysterious octagon of the medieval castle - Castel del Monte - in Andria, listed as a world interest site and the steppes with their orchids and hawks) were among the reasons for the public initiative of environmental preservation.

Remotely sensed satellite observations from space have fundamentally changed the way in which scientists study the atmosphere, oceans, land, vegetation, glaciers, sea ice and other environmental aspects of the earth's surface. In the past, the only way to investigate a given area was through direct observation and sampling on the ground and from ships or aircrafts [6]. Many portions of the globe are inaccessible and hardly known. Observations were mainly limited to those features that could be seen, photographed or measured in the visible portions of the spectrum. Local studies were compiled to provide regional interpretations of environmental conditions. However, it was difficult, if not impossible, to obtain global interpretations or to document changing conditions over large areas.

The archives of remotely sensed data acquired by earth resources satellite systems over the past three decades have provided an unprecedented data set for the study of land cover transformation. However, people interested in acquiring information on land cover and its transformation using remotely sensed data must consider a number of issues, not least the identification of the most appropriate data set(s) for the task in hand [7].

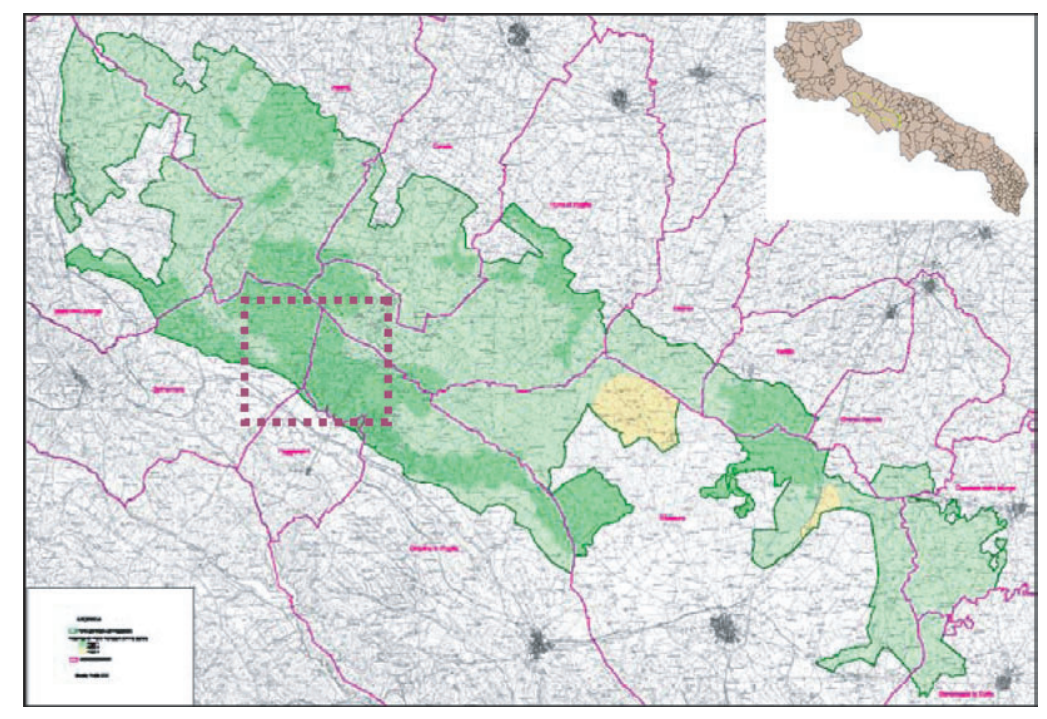

Figure 1: The Alta Murgia National Park in the Apulia region (Italy). 
The advent of the recent VHR satellite imagery has increased the possibility of investigating largescale areas in great detail. Such data are not a panacea for all applications, however, owing to a number of issues that arise with their use [8].

First of all, the very fine spatial and radiometric resolution of new generation satellite sensors is not always associated with high spectral resolution. For example, the increased textural information available in fine-resolution imagery allows for improved interpretation based on the shape and texture of the ground features, but techniques that have been developed to process and analyze current satellite data, including vegetation indices or multitemporal classification techniques that utilize mid-infrared or thermal channels, may not be applicable to the additional information provided by high-resolution satellites. The solutions suggested for solving this problem revolve around the use of various methods of data fusion, by merging the higher resolution panchromatic data with the lower resolution multispectral data [9]. Additionally, one may observe that the fusion issue is, and remains, of prime importance in diachronic analyses using images recorded by different generation and typology sensors (i.e. with different spatial resolutions).

Other issues include difficulties in acquiring consistent sequential acquisition dates, intensive computer processing time and disk space required to store larger image data sets and considerations of economic efficiencies [10]. Moreover, an improved scale of investigation implies the complexity of ground features [11] and can make visual interpretations of satellite imagery both labor intensive and uncertain. Lower resolution data are not greatly affected by artifacts, such as shadows, and also 'smooth' out variations across ranges of individual pixels, allowing statistical processing to create effective land cover maps.

In this paper, an object-oriented classification methodology on IKONOS multispectral data was implemented on the test area of the Alta Murgia National Park in the Apulia region (Italy).

The identification of the study areas, in which the rock-breaking phenomena for land cover transformation are present, was conducted with a classification strategy that was able to distinguish land use functions on the basis of differences in spatial distribution and pattern of land cover forms.

It consists of two phases: segmentation of the image into meaningful multipixel objects of various sizes, based on both spectral and spatial characteristics of groups of pixels; then, assignment of the segments (objects) to the classes using fuzzy logic and a hierarchical decision key.

\section{THE EXTRACTION OF THE INFORMATION ON LAND COVER FROM VHR SATELLITE IMAGERY}

Remotely sensed satellite data and images of the earth have the following four important advantages compared to ground observations:

1. Synoptic view: satellite images are 'big-picture' views of large areas of the surface. The position, distribution and spatial relationships of features are clearly evident; patterns within landscapes, seascapes and icescapes are emphasized. The major biologic, tectonic, hydrologic and geomorphic factors stand out distinctly.

2. Repetitive coverage: repeated images of the same regions, taken at regular intervals over periods of days, years and decades, provide data bases for recognizing and measuring environmental changes. This is crucial for understanding where, when and how the modern environment is changing.

3. Multispectral data: satellite sensors are designed to operate in many different portions of the electromagnetic spectrum utilizing atmospheric windows. Ultraviolet, visible, infrared and microwave energy coming from the earth's surface or atmosphere contain a wealth of information about material composition and physical conditions. 
4. Low-cost data: near-global repetitive collection of data is far cheaper using satellite sensors than it would be through conventional ground surveys [12].

Currently, remotely sensed data for the study of regional and global scale environmental changes are available from a multitude of sensors. Each has its own intrinsic characteristics [13, 14] and so the choice becomes more challenging since these characteristics determine the suitability of the data set for any particular task.

Once a data set has been identified, the method of extracting the information on land cover and its transformations must be considered because the accuracy of the final data processed may depend on the method used.

In remote sensing techniques, the informational classes of thematic mapping are not directly registered, but must be derived indirectly by using evidence contained in the spectral data of an image. Many approaches are available. Commonly, techniques that include a range of statistical, structural and neural approaches are used. Integrated strategies of classification are particularly useful, especially when information on land cover transformation is necessary in order to evaluate the effects of these processes and to provide one of the information layers needed for designing national environmental strategies.

When standard procedures of per-pixel multispectral classification are applied to VHR data, the increase of spatial resolution leads to augmentation in the ambiguity in the statistical definition of land cover classes and a decrease in the accuracy in automatic identification. These imagery sources are likely to generate other problems. Even if the radiometric resolution is enhanced (11 bits for IKONOS or QuickBird imagery), the spectral capabilities are generally limited compared to those of the previous generation sensors (seven bands for Landsat TM) [11]. Moreover, together with an increase in the spatial resolution, there is usually an increase in the variability within land parcels ('noise' in the image), generating a decrease in the accuracy of land use classification on a per-pixel basis [15].

In order to solve such problems, some post-classification procedures were investigated on the basis of intrinsic contextual data information [16, 17]. Although a reduction of noise in the classified image was obtained, substantial improvement in the overall accuracy was not seen. Moreover, a loss of meaningful information in classified data was noticed because of geometric and dimensional noncorrespondence of real elements with the moving window implementation matrix (e.g. with majority logical filter). Such methods need extensive editing operations on classified images in order to be stored in GIS databases [18].

An alternative technique to per-pixel classification is the per-field classification (so called because fields, as opposed to pixels, are classified as independent units), which takes into account spectral and spatial properties of the imagery, size and shape of the fields and the land cover classes chosen. In fact, this approach requires a priori information on the boundaries of objects in the image. 'Field' or 'parcel' refers to homogeneous patches of land (agricultural fields, gardens, urban structures or roads), which already exist and are superimposed on the image. Some studies $[19,20]$ indicate that this methodology contributes positively to the classification of remote sensing imagery of high to medium geometric resolution.

Problems arise in cases where no boundaries are readily available or when those boundaries should be updated [21]. One solution is image segmentation. In many cases, image analysis leads to meaningful objects only when the image is segmented in 'homogeneous' areas [22, 23]. Because an 'ideal' object scale does not exist, objects from different levels of segmentation (spatially) and with different meanings have to be combined for many applications. Our eyes recognize large and small objects simultaneously but does not cross totally different dimensions. From a balloon for instance, the impression of a landscape is dominated by land use patterns such as the composition of fields, roads, ponds/lakes and built-up areas. Closer to the ground, one begins to recognize small patterns such 
as single plants, while simultaneously small-scale patterns lose importance or cannot be perceived anymore. In remote sensing, a single sensor highly correlates with a specific range of scales [24].

The recognition of an object can be treated according to the sensor's resolution. A coarse rule of thumb is that the scale of the image objects to be detected must be significantly bigger than the scale of the image noise relative to texture. This ensures that subsequent object-oriented image processing is based on meaningful image objects. Therefore, homogeneity of the objects is one of the most important characteristics of a segmentation procedure [25]. The resulting segmentation should be reproducible and universal, thus allowing the application to a large variety of data $[22,26]$. Moreover, multiresolution image processing based on texture and utilizing fractal algorithms can alone fulfill all the main requirements at once. Their 'fractal net evolution approach' uses a local mutual best-fit heuristics to find the least heterogeneous merge in a local vicinity, following the gradient of the best-fit. Furthermore, their algorithm can be applied with pure spectral heterogeneity or with a mix of spectral and form heterogeneity [21].

\section{DATA AND METHODS}

The study area of this research is in Italy, in the region of Apulia, in a part of an area of Alta Murgia National Park, where the custom of crushing rock and stones for soil adaptation in agriculture has been practiced over the last 20 years.

The data sources acquired for the analysis consisted of:

- IKONOS multispectral images (Acquisition Date/Time: May 18, 2000/09:33): IKONOS is the first commercially owned satellite providing $1 \mathrm{~m}$ resolution panchromatic image data and $4 \mathrm{~m}$ multispectral imagery [27]. The multispectral image data include three visible and one infrared channel. Data are collected in 11-bit radiometric resolution and provided in a format compatible with the image analysis software. The tile size for each individual scene is $11.3 \times 11.3 \mathrm{~km}$. Because of its pointable off-nadir viewing capability, the satellite revisit interval is as little as 3-4 days. To reduce the size of the image to be manipulated and, to maintain a geographic consistency during the analysis, equivalent sub-scenes were defined (Fig. 2).

- Training set and ground reference data obtained by land use survey and additional maps.

\subsection{Classification strategy}

Object-oriented image analysis consists of two steps: segmentation and (fuzzy) classification of the resulting image objects [25]. The multiresolution segmentation algorithm implemented in eCognition software extracts homogeneous image objects at different resolution hierarchy levels respectively. For each segment (object) object features are calculated and stored in a database. The next step of the process is fuzzy classification. It is based on the different object features available. In contrast to pixel-based statistic classifiers, fuzzy classification replaces the strict 'yes/no' class definitions by a continuous range where all the numbers between 0 and 1 describe a certain state of class membership [21].

An important aspect to understand the content of an image is context. There are two types of contextual information: environmental, which describes the situation of the image (basically time, sensor and location) and local context, which describes the relationships of objects to each other within a certain area of the image - usually neighborhood relationships.

In order to model spatial context, it is necessary to link the different sized image objects hierarchically and to represent their (semantic) scale relationships. From a classification point of view, the non-intrinsic properties, such as neighborhood relationships or being a sub-object or a super-object, 


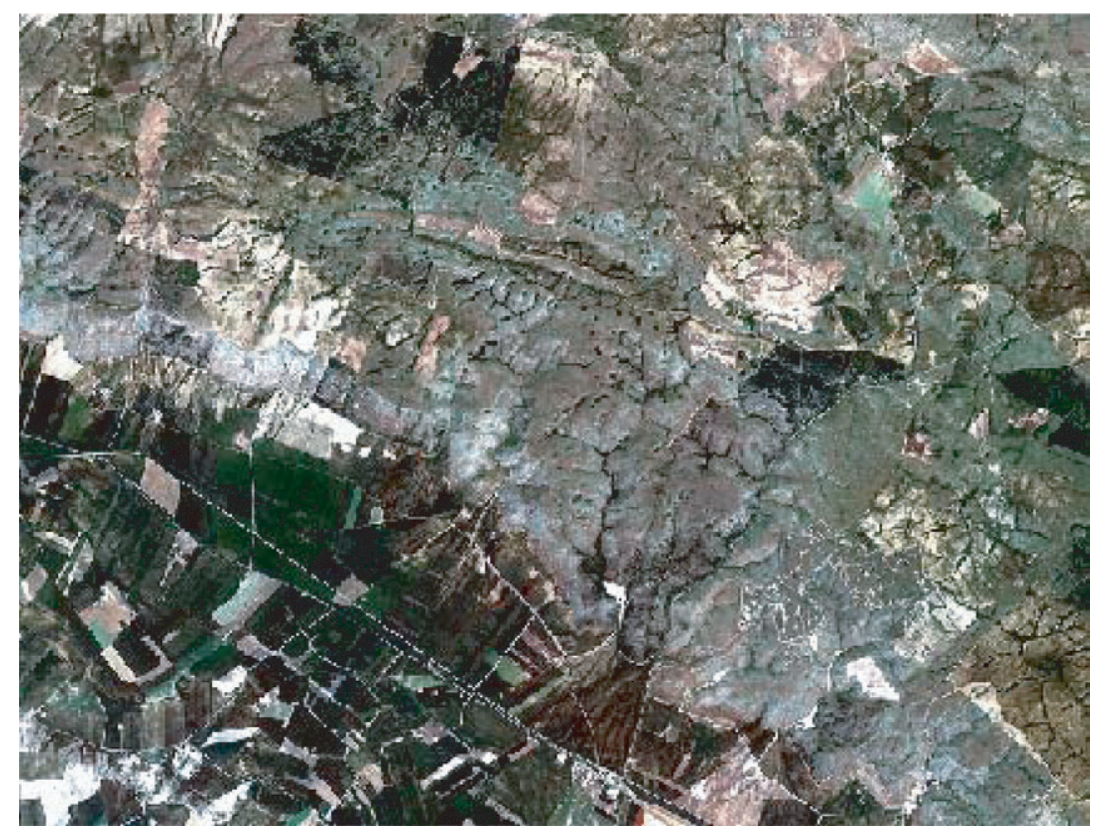

Figure 2: IKONOS multispectral image processed in RGB mode.

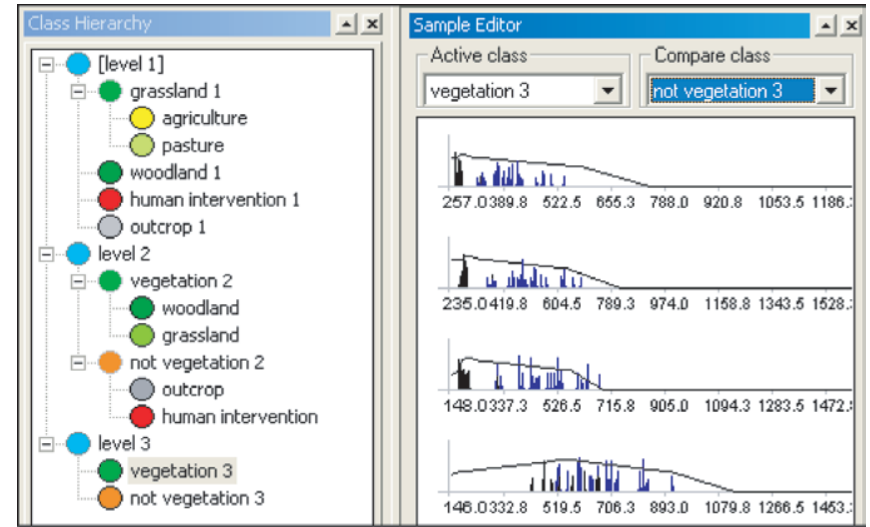

Figure 3: Hierarchical relations and samples analysis.

are describable. Figure 3 shows the semantic structure, adopted in this study, based on hierarchical relations between the different segmentation levels.

The strategy for the classification was multiresolution segmentation, which uses information provided by small object primitives to label large scale objects. Such algorithms join neighboring regions that show a degree of fitting - computed with respect to their spectral variance and/or their shape properties - which is smaller than a predefined threshold (scale parameter). The choice of this parameter determines the number and size of the resulting segments [24]. The selection of scale parameters was purely empiric. The primitive objects in a 30-scale, generates bigger objects in a 60-scale and so on (Fig. 4). 


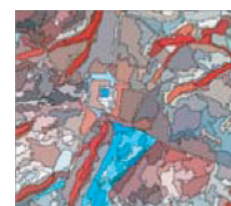

Level 1 - 30

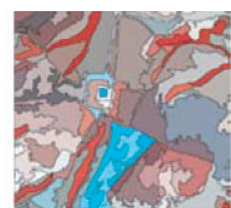

Level 2 - 60

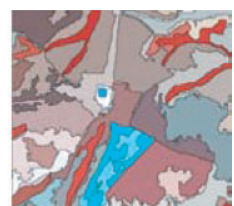

Level 3 - 90

Figure 4: Image segmentation using three different scale parameters.

The classes are described using spectral, texture and shape information. Some parameters for the membership functions used to classify on different scale levels are explained as follows:

- brightness (the sum of the mean values containing spectral information divided by their quantity);

- mean difference to scene (the difference between the layer mean value and the mean value of the whole scene);

- GLCM Homogeneity (the local homogeneity of the gray level co-occurrence matrix of an image);

- density (the area covered by the image object divided by its radius).

Generally in higher hierarchy, spectral information yields good results, such as mean brightness and ratio. To distinguish different objects, which are very close spectrally, form parameters such as density, asymmetry and shape index could be useful.

The features concerning the texture are based on sub-object analysis. The texture features might be related to spectral information or spatial information of the sub-objects [26]. A fuzzy rule can have one single condition or can consist of a combination of several conditions, which have to be fulfilled for an object to be assigned to a class. In case of vegetation classification, the spectral information of the near-infrared channel can play an important role. On the other hand, parameters such as shape, index and asymmetry can be used to discriminate objects that differ in shape. Our classification was based on specialization, that means, starting with bigger objects (the first segmented image) few classes could be depicted, i.e. vegetation and not vegetation and these classes could be divided into sub-classes in the smaller segments, i.e. agriculture and pasture, according to the semantic net. The purpose is to deliver basic information for the classification of level 3 to level 2 and finally to level 1 with smaller objects (Fig. 5). The process of subdividing parent classes into child sub-classes might take supplementary data into account. The links between objects and layers can be used to formulate a class decision rule.

The territory analyzed includes four main semantic groups of land use (grassland, woodland, outcrops and human interventions), which include low-level objects. The definition of classes is based on the possibility of distinguishing one class from another in certain features. This is realized by developing a knowledge base suitable for each classification strategy enabling the description of each class. In order to assess the capability to discriminate human intervention features in natural areas, level 1 for the classification was selected. Classes separated by object features can represent parts of a thematic unit and can be combined into semantic groups. Generally, remotely sensed reflectance is related to land cover and not to land use, but in the present case each land use class was assumed to correspond to spectrally separable land covers. After this, the nearest neighbor (NN) classifier was trained on the representative sample of each of the land use classes.

\section{RESULTS}

The presented classification results show the ability of the so far developed rule sets to distinguish between objects and to generate semantic classes. This is demonstrated by the value of the overall accuracy (0.78) of the classification as shown in Table 1. 


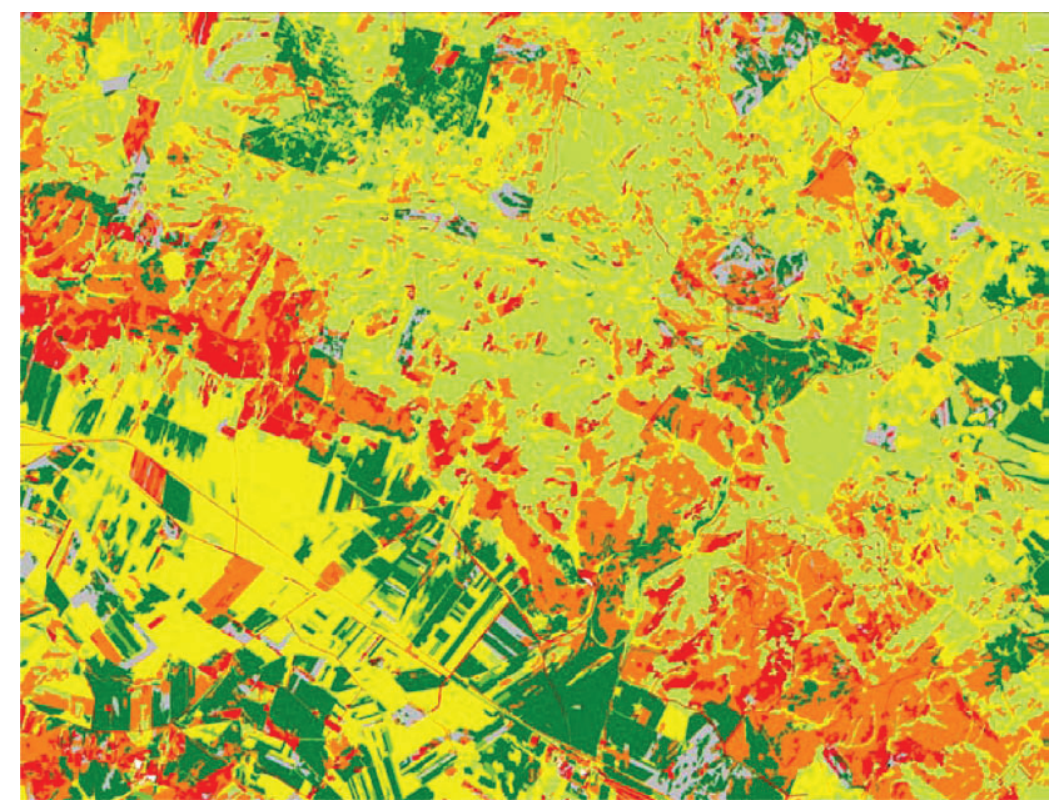

Figure 5: Classification (level 1).

Table 1: Accuracy of classification.

\begin{tabular}{lc}
\hline Classes & Assessment \\
\hline Woodland & 0.85 \\
Agriculture & 0.73 \\
Pasture & 0.86 \\
Human intervention & 0.76 \\
Outcrop & 0.68 \\
Overall accuracy & 0.78 \\
\hline
\end{tabular}

There are various causes for obvious 'classification' errors with relevant difficulty in distinguishing between different natural and artificial areas. For example, outcrop shows similar characteristics to other classes imputed to the presence of vegetation and their different orientation towards the sunlight. These properties caused intense overlaps with human intervention and pasture.

For spectral separable classes, such as natural features, like woodland, a good recognition rate was obtained.

Semantic classes that present errors often contain different class types causing a partly incorrect representation. For example a class for agriculture can include trees or grass. Object-oriented classification distinguishes between these objects and classifies trees or grass (as pasture) depending on the object level.

\section{CONCLUSIONS}

The semantic classes derived from satellite data cannot exactly fit the classes for large-scale investigation because of the resulting aggregation of information. 
Very high-resolution satellite data contain significantly more detailed information than previous satellites (e.g. Landsat TM) but problems arise from their high spectral variance. The so-called 'salt \& pepper' effect is a handicap for the recognition of semantic classes.

Object-oriented classification systems enable us to analyze high-resolution data and avoid some of the above-mentioned problems. Based on a preceding segmentation, a fuzzy approach of nearest neighbor is used to classify the IKONOS data.

The primary task is the creation of object lists that take into account the abilities of different remote sensing data. Major problems are the generation of meaningful objects that fit semantic classes of the object lists.

Classification rules for all object types and their representation in VHR data from different sensors and on varying acquisition times need to be developed.

The next step will be the assignment of the deduced classification rules on more complex specializations and hierarchical networks of classes. Additional knowledge from thematic layers has not been used in this study so far but will be included in our future work.

\section{REFERENCES}

[1] Basso, F., Bove, E., Dumontet, S., Ferrara, A., Pisante, M., Quaranta, G. \& Taberner, M., Evaluating environmental sensitivity at the basin scale through the use of geographic information systems and remotely sensed data: an example covering the Agri basin Southern Italy. Catena, 40, pp. 19-35, 2000.

[2] Thornes, J.B., Mediterranean desertification and the vegetation cover. EUR 15415 - Desertification in a European Context: Physical and Socio-economic Aspects, eds. R. Fantechi, D. Peter, P. Balabanis \& J.L. Rubio, Office for Official Publications of the European Communities: Brussels and Luxembourg, pp. 169-194, 1995.

[3] UNEP (United Nations Environmental Programme), Draft plan of actions to combat desertification. UN Conference on Desertification, Nairobi, 1977.

[4] UNEP (United Nations Environmental Programme), World Atlas of Desertification, Edward Arnold: Seven Oaks, UK, 1992.

[5] Polytechnic University of Bari, Italy (scientific coordination by Borri D.), Alta Murgia National Park, Final Report, 2002.

[6] DeFries, R.S. \& Townshend, J.R.G., Global land cover characterisation from satellite data: from research to operational implementation. Global Ecology and Biogeography, 8, pp. 367-379, 1999.

[7] Franklin, S.E. \& Wulder, M.A., Remote sensing methods in medium spatial resolution satellite: land cover classification of larges areas. Progress in Physical Geography, 26, pp. 173-205, 2002.

[8] Goetz, S.J., Wright, R.K., Smith, A.J., Zinecker, E. \& Schaubb, E., IKONOS imagery for resource management: tree cover, impervious surfaces, and riparian buffer analyses in the midAtlantic region. Remote Sensing of Environment, 88, pp. 194-208, 2003.

[9] Pohl, C. \& Van Genderen, J.L., Multisensor image fusion in remote sensing: concepts, methods and applications. International Journal of Remote Sensing, 19(5), pp. 823-854, 1998.

[10] Fisher, J. \& Goetz, S.J., Considerations in the use of high spatial resolution imagery: an applications research assessment. ASPRS Conference Proceedings, St. Louis, MO, Available from ASPRS, http://www.asprs.org and at http://www.geog.umd.edu/resac, 2001.

[11] Fritz, L.W., High resolution commercial remote sensing satellites and spatial information. http://www.isprs.org/publications/highlights/highlights0402/fritz.html, 1999. 
[12] Verstraete, M., The contribution of remote sensing to monitor vegetation and to evaluate its dynamic aspects. Vegetation, Modeling and Climatic Change Effects, eds. F. Veroustraete, R. Ceulemans, SPB Academic Publishing: The Hague, Netherlands, pp. 207-212, 1994.

[13] Ehlers, M., Jadkowski, M.A., Howard, R.R. \& Brostuen, D.E., Application of SPOT data for regional growth analysis and local planning. Photogrammetric Engineering and Remote Sensing, 56(2), pp. 175-180, 1990.

[14] Lillesand, T.M. \& Kiefer, R.W. (eds.), Remote Sensing and Image Interpretation, 4th edn., Wiley \& Sons: New York, 2000.

[15] Woodcock, C. \& Strahler, A., The factor of scale in remote sensing. Remote Sensing of Environment, 21, pp. 311-322, 1987.

[16] Townshend, J.R.G., The enhancement of computer classification by logical smoothing. Photogrammetric Engineering and Remote Sensing, 52, pp. 213-221, 1986.

[17] Townshend, J.R.G., Land cover, International Journal of Remote Sensing, 13, pp. 1319-1328, 1992.

[18] Donnay, J.P., Use of remote sensing information in planning. Geographical Information and Planning, eds. J. Stillwell, S. Geertman \& S. Openshaw, Springer-Verlag: Berlin, pp. 242-260, 1999.

[19] Aplin, P., Atkinson, P. \& Curran, P., Per-field classification of land use using the forthcoming very fine resolution satellite sensors: problems and potential solutions. Advances in remote sensing and GIS analysis, eds. P. Atkinson \& N. Tate, Wiley \& Son: Chichester, pp. 219-239, 1999.

[20] Caprioli, M. \& Tarantino, E., Accuracy assessment of per-field classification integrating very fine spatial resolution satellite sensors imagery with topographic data. Journal of Geospatial Engineering, 3(2), pp. 127-134, 2001.

[21] Blaschke, T., Lang, S., Lorup, E., Strobl, J. \& Zeil, P., Object-oriented image processing in an integrated GIS/remote sensing environment and perspectives for environmental applications. Environmental Information for Planning, Politics and the Public, Vol. 2, eds. A. Cremers, K. Greve, Metropolis Verlag: Marburg, pp. 555-570, 2000.

[22] Baatz, M. \& Schäpe, A., Multiresolution segmentation - an optimisation approach for high quality multi-scale image segmentation. Angewandte Geographische Informationsverarbeitung, Vol. XII, eds. J. Strobl, Blaschke, Griesebner, Wichmann-Verlag: Heidelberg, pp. 12-23, 2000.

[23] Gorte, B., Probabilistic Segmentation of Remotely Sensed Images. ITC Publication Series, 63, 1998.

[24] Schiewe, J., Tufte, L. \& Ehlers, M., Potential and problems of multi-scale segmentation methods in remote sensing. Geo-Informations-Systeme, 6, pp. 34-39, 2001.

[25] Baatz, M., Heynen, M., Hofmann, P., Lingenfelder, I., Mimler, M., Schäpe, A., Weber, M. \& Willhauck, G., eCognition User Guide, Definiens AG: München, 2000.

[26] Antunes, A.F.B., Lingnau, C. \& Da Silva, J.C., Object oriented analysis and semantic network for high resolution image classification. Proc. of Anais XI SBSR, Belo Horizonte, Brasil, INPE, pp. 273-279, April 05-10, 2003.

[27] Dial, G.F., Bowen, H., Gerlach, B., Grodecki, J. \& Oleszczuk, R., IKONOS satellite sensor, imagery and products. Remote Sensing of Environment, 88, pp. 23-36, 2003. 